\title{
Enhanced Potential of Therapeutic Applications of Curcumin Using Solid-in-Water Nanodispersion Technique
}

\author{
Safrina Dyah Hardiningtyas ${ }^{1}$, Rie Wakabayashi ${ }^{1}$, \\ Ryutaro IsHIYAmA $^{1}$, Yuki OWADA ${ }^{1}$, Masahiro Goto ${ }^{1,2}$ and \\ Noriho KAMIYA ${ }^{1,2}$ \\ ${ }^{1}$ Department of Applied Chemistry, Graduate School of Engineering, Kyushu University, \\ Motooka 744, Nishi-ku, Fukuoka-shi, Fukuoka 819-0395, Japan \\ ${ }^{2}$ Division of Biotechnology, Center for Future Chemistry, Kyushu University, \\ Motooka 744, Nishi-ku, Fukuoka-shi, Fukuoka 819-0395, Japan
}

\begin{abstract}
Keywords: Curcumin, Solid-in-Water Nanodispersion, Anticancer Activity
Curcumin (Cur), a hydrophobic polyphenol compound, holds promising potential as an anticancer agent. However, the poor solubility in water and the low bioavailability of curcumin have limited its therapeutic applications. In this regard, we reported the formulation of curcumin using a solid-in-water $(\mathrm{S} / \mathrm{W})$ nanodispersion technique to enhance the water solubility and therapeutic activity of curcumin. This new aqueous formulation comprises simple preparation protocols: emulsification and freeze-drying for encapsulating hydrophobic biomolecules with a hydrophilic surfactant, followed by redispersion of the resultant solid complexes in an aqueous solution. Pluronics F68 and F127 were used here for the encapsulation of curcumin. Enhanced aqueous solubility of curcumin was achieved by encapsulating curcumin with a hydrophilic surfactant using the $\mathrm{S} / \mathrm{W}$ nanodispersion technique. The resultant nanosized formulation had a narrow size distribution and high entrapment efficiency of curcumin. The highest loading capacity of curcumin in S/W nanodispersion was obtained with a weight ratio of curcumin to pluronic of $1: 10$ for both surfactants. The release profile of the complexes was found to depend on the type of surfactant, suggesting that the selection of a proper surfactant is crucial for controlling curcumin delivery. The anticancer activity of the $\mathrm{S} / \mathrm{W}$ formulation of curcumin was correlated with the drug release profiles and cellular uptake, which in turn was influenced by the hydrophobicity and chemical structure of the surfactant.
\end{abstract}

\section{Introduction}

Cancer is a severe disease with high mortality, accounting for 8.8 million deaths worldwide (about $17 \%$ of all deaths) in 2015 (WHO, 2017). Polyphenol compounds are natural products that have shown promising anticancer activities. Diferuloylmethane or 1,7-bis(4-hydroxy3-methoxyphenyl)-1,6-heptadiene-3,5-dione, also known as curcumin, is a hydrophobic polyphenolic compound derived from the rhizome of the plant Curcuma longa L. Curcumin has a low molecular weight and is potentially a promising compound for cancer prevention and treatment (Anand et al., 2007; Gupta et al., 2013). In vivo studies have shown that curcumin suppresses the proliferation of various types of cancer cells, such as breast cancer (Bayet-Robert et al., 2010), colon cancer (Yang et al., 2015) and pancreatic cancer (Li et al., 2005). Curcumin also has multiple pharmacological activities, including antibacterial, antioxidant, antiinflammatory (Anand et al., 2007), and antiviral activities (Mathew and Hsu, 2018). Clinical trials have shown limited toxicity of curcumin even at very high doses of $12 \mathrm{~g} / \mathrm{d}$ (Lao

Received on February 23, 2018; accepted on April 22, 2018

DOI: $10.1252 /$ jcej.18we 060

Correspondence concerning this article should be addressed to $\mathrm{N}$. Kamiya (E-mail address: kamiya.noriho.367@m.kyushu-u.ac.jp). et al., 2006). Despite the favorable pharmacodynamics and toxicity profiles, its therapeutic applications are restricted by its extremely low bioavailability and poor absorption. In two studies, little or no curcumin was detected in serum after oral or intravenous administration (Chen et al., 2016; Lao et al., 2006). The low bioavailability of curcumin is attributed to its very low aqueous solubility at neutral or acidic $\mathrm{pH}$, where the solubility of curcumin in a buffer of $\mathrm{pH} 5$ is reportedly $3.8 \times 10^{-8} \mathrm{M}$ (Tønnesen et al., 2002).

Over the past decade, there has been increasing development of formulation technology that aims to improve the solubility and pharmacokinetics of curcumin. Nanocarriers are among the most promising drug delivery systems for realizing curcumin's potential as a preventive or therapeutic agent. A number of nanocarrier systems loaded with curcumin have been reported to date, such as liposomes (Li et al., 2005), polymer micelles (Gong et al., 2013; Yang et al., 2015), chitosan nanoparticles (Razi et al., 2018a, 2018b) and nanoemulsions (Sari et al., 2015). In the present study, we aimed to improve the solubility and bioavailability of curcumin by developing a new aqueous formulation using the solid-in-water (S/W) nanodispersion technique (Wakabayashi et al., 2014). This novel technique comprises simple preparation protocols: emulsification and freeze-drying for encapsulating hydrophobic biomolecules with a hydrophilic surfactant, followed by redispersion of the resultant solid complexes in an aqueous 
solution. Our previous studies have demonstrated that this technique enables the encapsulation of the poorly soluble anticancer drug camptothecin (CPT) with high efficiency and stability (Wakabayashi et al., 2014). Here, we applied this formulation strategy to study the nanodispersion of curcumin in the aqueous phase and characterize the $\mathrm{S} / \mathrm{W}$ nanodispersion in detail.

\section{Experimental}

\subsection{Materials}

Curcumin was purchased from Wako Pure Chemical Industries, Ltd. Pluronic F68 and F127 were purchased from MP Biomedical, Inc. and Sigma-Aldrich Co., respectively. Rhodamine-labelled dioleyl phosphatidylcholine (RhoDOPE) was purchased from Polar Lipid, Inc. HeLa cell lines were purchased from RIKEN Cell Bank and were cultured in minimum essential medium (MEM) supplemented with $10 \%$ fetal bovine serum (FBS) and $1 \%$ of antibiotic-antimycotic mixed stock (Nacalai Tesque, Inc.). All cells were incubated at $37^{\circ} \mathrm{C}$ with humidified $5 \% \mathrm{CO}_{2}$ atmosphere.

OPTI-MEM was purchased from Thermo Fisher Scientific, Inc. Cell counting kit-8 was purchased from Dojindo Laboratories. Dulbecco's phosphate-buffered saline (D-PBS) was purchased from Nacalai Tesque, Inc. All other chemicals were of analytical grade.

\subsection{Preparation of curcumin loading solid-in-water nanodispersion}

Briefly, curcumin solution was prepared by first dissolving curcumin in acetone to a concentration of $4 \mathrm{mg} / \mathrm{mL}$. Curcumin in acetone $(0.05 \mathrm{~mL})$ was added to cyclohexane $(0.95 \mathrm{~mL})$. Then, $1 \mathrm{~mL}$ of the curcumin solution and $4 \mathrm{~mL}$ of an aqueous solution of Pluronic F68 or F127 (0.5 or $1 \mathrm{mg} / \mathrm{mL}$ ) were mixed using a homogenizer (Polytron ${ }^{\circledR}, \mathrm{Ki}-$ nematica, Inc.) at 26,000 rpm for $90 \mathrm{~s}$ to form an oil-in-water emulsion. The emulsion was immediately frozen in liquid nitrogen for $20 \mathrm{~min}$ and lyophilized overnight using a freeze dryer (EYELA, Ltd.) to obtain curcumin-pluronic complexes. The resultant complexes were dispersed in PBS ( $\mathrm{pH} 7.4$ ) to the final concentration of $0.1 \mathrm{mg} / \mathrm{mL}$, and this solution was employed as a curcumin-solid-in-water nanodispersion (Cur-S/W nanodispersion). The preparation procedure of

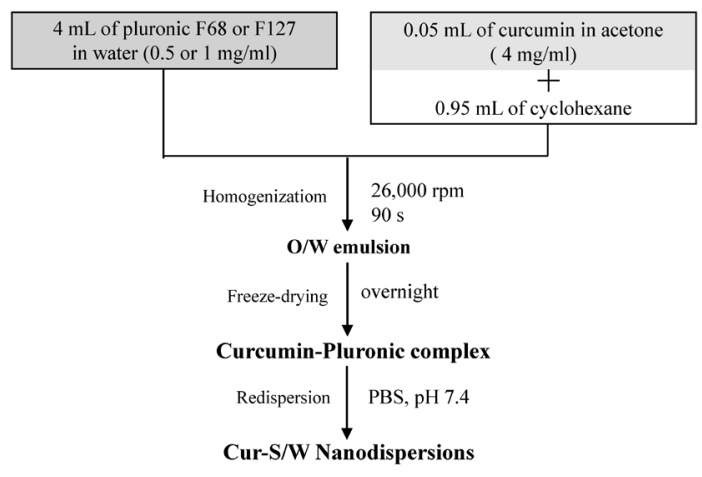

Fig. 1 Preparation procedure of Cur-S/W nanodispersions
Cur-S/W nanodispersions is illustrated in Figure 1.

\subsection{Characterization of Cur-S/W nanodispersions}

The size distribution and polydispersity index of Cur-S/W nanodispersions were determined using dynamic light scattering (DLS) at $25^{\circ} \mathrm{C}$ at a detection angle of $173^{\circ}$ with 10 measurement cycles per run (Zetasizer Nano-ZS, Malvern Instruments, Ltd.). The zeta potential was obtained using the same instrument. The measurement was performed in triplicate.

The encapsulation efficiency of Cur-S/W nanodispersions was determined by the syringe filtration method. The Cur-S/W nanodispersion was filtrated using a filter unit with pore size of $0.45 \mu \mathrm{m}$ (Avatec ${ }^{\circledR}$, Toyo Roshi Kaisha, Ltd.) to remove free curcumin. The concentration of curcumin was estimated by a reverse-phase high performance liquid chromatography (HPLC) unit equipped with an auto sampler (Jasco, LC-4000, Jasco Inc.). The samples were diluted 2-fold using acetonitrile before injection. In an amount of $20 \mu \mathrm{L}$, the sample was analyzed by a $\mathrm{C}_{18}$ column $\left(4.6 \times 250 \mathrm{~mm}\right.$, InertSustain $\left.{ }^{\circledR} \mathrm{C}_{18}\right)$ with a mobile phase of acetonitrile containing $0.1 \%$ trifluoroacetic acid $(55 \%$ $\mathrm{v} / \mathrm{v}$ ) and milli-Q-water at a flow rate of $0.8 \mathrm{~mL} / \mathrm{min}$. The encapsulation efficiency (EE) and loading capacity (LC) of curcumin in S/W nanodispersions were calculated using the equations below.

$$
\begin{aligned}
& \mathrm{EE}[\%]=\mathrm{Ws} / \mathrm{Wo} \times 100 \\
& \mathrm{LC}[\%]=\mathrm{Ws} /(\mathrm{Wp}+\mathrm{Wo}) \times 100
\end{aligned}
$$

Herein, Ws is the curcumin weight in Cur-Pluronic complex, Wo is the weight of the initial feeding drug, and Wp is the weight of the initial feeding pluronic.

The morphology of particles was observed by transmission electron microscopy (TEM-2010, JEOL, Ltd.). The Cur-S/W nanodispersions were directly deposited in a copper TEM grid with a carbon support and negatively stained with a $2 \%$ uranyl acetate solution. The excess solution was absorbed by filter paper and the grid was allowed to air-dry, and then kept in a desiccator for subsequent microscopy.

\subsection{In vitro release profile of curcumin from S/W nanodispersions}

The release of free Cur and Cur-S/W nanodispersions was evaluated using the dialysis bag method under physiological conditions (Chang et al., 2017). The Cur-S/W $(0.1 \mathrm{mg} / \mathrm{mL})$ was placed in a dialysis bag with $12-14 \mathrm{kDa}$ molecular weight cut-off (Spectra/Por ${ }^{\circledR}$, Spectrum Laboratories, Inc.). It was incubated at $37^{\circ} \mathrm{C}$ with equal volumes of PBS ( $\mathrm{pH} 7.4$ ) and ethanol as the release medium with sink condition, and shaken at $100 \mathrm{rpm}$. The control experiment was performed on curcumin solution with $10 \%$ dimethyl sulfoxide (DMSO) in PBS, which was placed in a dialysis bag and incubated under the same conditions. At each designated time point, $0.5 \mathrm{~mL}$ of the release medium was collected, and then replaced with the fresh release medium. The amount of curcumin in the release medium was quantified 
by HPLC as described previously.

\subsection{In vitro cytotoxicity studies}

The in vitro cytotoxicity of free Cur, Cur-S/W nanodispersion and the carrier (pluronic) was evaluated in HeLa cell lines. Free Cur was dissolved in DMSO to yield a final concentration of less than $0.5 \%$. HeLa cells were seeded at a density of 5000 cells in a 96-well plate (Greiner Bio-One $\mathrm{GmbH}$ ) and incubated overnight. The medium was exposed to a series of free Cur, Cur-S/W nanodispersion and blank $\mathrm{S} / \mathrm{W}$ nanodispersion in reduced serum medium (OPTIMEM, GIBCO) at different concentrations. Cells were incubated with each treatment for $24 \mathrm{~h}$. The samples were removed, and the cells washed with D-PBS (Nacalai Tesque, Inc.), then $100 \mu \mathrm{L}$ of WST-8 cell counting solution (Dojindo Molecular Technologies, Inc.) in OPTI-MEM was added to each cell, followed by incubation for $3 \mathrm{~h}$. The absorbance derived from WST-8 formazan was recorded on a microplate reader (Bio-Tek Instrument, Inc.) at $450 \mathrm{~nm}$. As the control, non-treated cells were incubated under the same conditions. Cell viability was expressed as (sample/control) $\times 100 \%$.

\subsection{Cellular internalization studies}

The Cur-S/W nanodispersions with Rho-DOPE were prepared as previously described (Wakabayashi et al., 2014), except that $10 \mu \mathrm{L}$ of $1 \mathrm{mg} / \mathrm{mL}$ Rho-DOPE in methanol was added to the pluronic aqueous solution. Cell internalization of Cur-S/W nanodispersion in HeLa cell was evaluated by confocal laser scanning microscopy (CLSM). The HeLa cells were seeded in a multi-well glass bottom dish at a density of 10,000 cells (Matsunami Glass Ind., Ltd.) and incubated overnight at $37^{\circ} \mathrm{C}$ under a $\mathrm{CO}_{2}$ atmosphere. The medium was removed, and $200 \mu \mathrm{L}$ of the samples dispersed in OPTIMEM containing $10 \mu \mathrm{g} / \mathrm{mL}$ of curcumin were added to the dish. After incubation for $3 \mathrm{~h}$, the samples were removed and fixation with $4 \%$ paraformaldehyde (Wako Pure Chemical Industries, Ltd.) was carried out for $10 \mathrm{~min}$ at $25^{\circ} \mathrm{C}$. A confocal image of HeLa cells was obtained using an LSM 700 confocal microscope (Carl Zeiss, Inc.). Curcumin exhibited green fluorescence, which was observed using the Fluorocein isothiocyanate (FITC) filter. The rhodamine filter was used to observe the red fluorescence of curcumin-surfactant complexes labelled with Rho-DOPE.

\section{Results and Discussion}

Curcumin is well known for its poor solubility in water and its instability. The $\mathrm{S} / \mathrm{W}$ nanodispersion technique is the encapsulation of hydrophobic drugs with hydrophilic surfactant by formation of an oil-water emulsion using a high-speed homogenizer, and subsequent freeze drying overnight to form the solid hydrophobic drug-surfactant complex. The complex was then redispersed in an aqueous solution to obtain a homogeneous nanodispersion. To validate the S/W technology as a delivery vehicle for curcumin, we used two different types of pluronics as surfactant to form curcumin-pluronic complexes. Pluronic is an A-B-A triblock copolymer with polymeric material A being a hydrophilic block poly(ethylene oxide) (PEO) and $\mathrm{B}$ being a hydrophobic block poly(propylene oxide) (PPO). It has been approved by the Food and Drug Administration (FDA) as an injectable material and is commercially available. Pluronics F68 and F127 were employed here for the encapsulation of curcumin.

\subsection{Characteristics of Cur-S/W nanodispersions}

Here, we used different types of pluronic block copolymers and investigated their effects on the properties of the Cur-S/W nanodispersions. These hydrophilic surfactants are nonionic surfactants with different lengths of hydrophilic EO and hydrophobic PO chains, where the composition of EO and PO for Pluronic F68 and F127 are $\mathrm{EO}_{76}-\mathrm{PO}_{29}-\mathrm{EO}_{79}$ and $\mathrm{EO}_{100}-\mathrm{PO}_{65}-\mathrm{EO}_{100}$, respectively (Sezgin et al., 2006). These varieties in the composition of EO and $\mathrm{PO}$ chains result in differences in the hydrophobicity of pluronics, and Pluronic F127 shows a higher hydrophobicity (Hydrophilic Lipophilic Balance value, HLB of 22) than F68 (HLB of 29). Curcumin was incorporated into the hydrophobic core of surfactant through the hydrophobic interaction of curcumin with the hydrophobic block PPO of pluronic. When the curcumin-pluronic complexes were dispersed in PBS, the difference in physical appearance of Cur-S/W nanodispersions prepared with the two types of surfactants is shown in Figure 2. The translucent dispersion was obtained by dispersing curcumin-pluronic complexes in PBS. In contrast, a physical mixture of curcumin in PBS formed a yellow precipitate.

The mean particle size of Cur-S/W nanodispersions prepared with different weight ratio of curcumin to pluronic was analyzed by DLS. The nanosize range of the Cur-pluronic complexes with narrow dispersities were obtained using the nanodispersion technique, as summarized in Table 1. The mean particle size of Cur-S/W nanodispersion with Cur-F127 complex (av. 27-28nm) was smaller than that of the Cur-F68 complex (av. 100-130 nm). In the present work, the Cur-S/W nanodispersions were prepared by lyophilization of cyclohexane-in-water emulsion with plu-

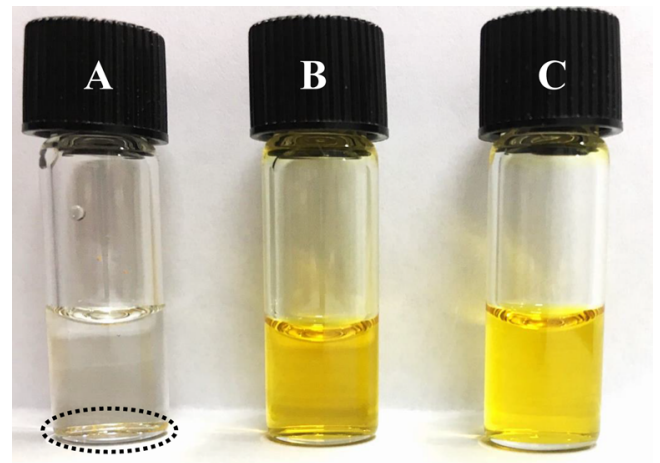

Fig. 2 Physical appearance of curcumin in PBS (A) and curcumin loaded S/W nanodispersions with Pluronic F68 (B) or Pluronic F127 (C) in PBS; weight ratio of curcumin to pluronic was $1: 10$; all samples contained $0.1 \mathrm{mg} / \mathrm{mL}$ of curcumin 
Table 1 Characteristics of Cur-S/W nanodispersions with two different surfactants

\begin{tabular}{|c|c|c|c|c|c|c|}
\hline Sample & Type of surfactant & $\begin{array}{c}\text { Weight ratio of } \\
\text { curcumin: surfactant }\end{array}$ & Z-size $[\mathrm{nm}]$ & PDI & $\mathrm{EE}[\%]$ & LC [\%] \\
\hline Cur-F68_\#1 & Pluronic F68 & $1: 20$ & $105 \pm 2$ & $0.09-0.12$ & $95 \pm 3$ & $4.4 \pm 0.4$ \\
\hline Cur-F68_\#2 & Pluronic F68 & $1: 10$ & $113 \pm 3$ & $0.07-0.11$ & $81 \pm 4$ & $7.3 \pm 0.1$ \\
\hline Cur-F127_\#1 & Pluronic F127 & $1: 20$ & $27 \pm 0.7$ & $0.2-0.26$ & $88 \pm 4$ & $4.2 \pm 0.4$ \\
\hline Cur-F127_\#2 & Pluronic F127 & $1: 10$ & $28 \pm 0.5$ & $0.2-0.23$ & $86 \pm 5$ & $7.9 \pm 0.2$ \\
\hline
\end{tabular}

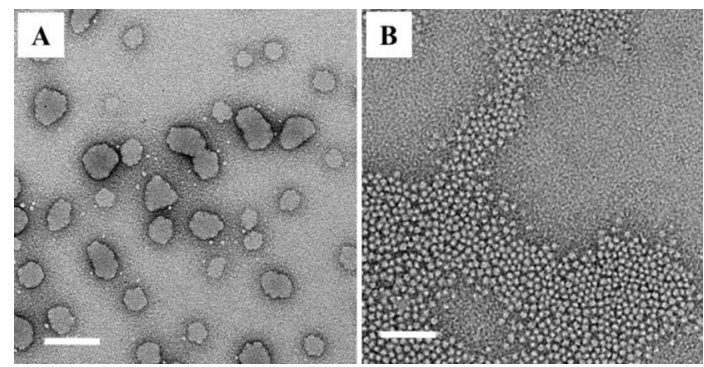

Fig. 3 TEM images of Cur-S/W nanodispersions prepared with Pluronic F68, Cur-F68_\#2 (A) and Pluronic F127, Cur-F127_\#2 (B). Scale bar: $200 \mathrm{~nm}$

ronic as a stabilizer. The hydrophobic surface of oil droplets was stabilized with the hydrophobic block PPO, while the two hydrophilic block PEO tails directed to the aqueous solution (Tadros, 2009). Therefore, the length of hydrophobic block PPO chain in polymer composition may have influenced the particle size of Cur-S/W nanodispersions.

The LC of curcumin in the formulations was also studied. The results in Table 1 suggest that a higher LC was obtained with a weight ratio of curcumin to pluronic of 1:10 for both surfactants (Cur-F68_\#2 and Cur-F127_\#2), and the conditions were selected for further characterization. When the loading concentration of curcumin was increased until the weight ratio of curcumin to pluronic reached $1: 5$, the complexes became extremely unstable, and the particle could not be characterized (data not shown). The increase in loading concentration of curcumin caused a notable increase in the particle size, as indicated in Table 1. In addition, the EE of Cur-S/W nanodispersion was evaluated by a filtration method using HPLC analysis. The results showed the quantitative EE of curcumin for all formulations prepared with the two types of surfactants and the different weight ratios of curcumin to pluronic (Table 1). High $\mathrm{EE}$ of curcumin was achieved by the $\mathrm{S} / \mathrm{W}$ nanodispersion technique, with approximately $81 \%$ EE for cur-F68_\#2 and $86 \%$ for cur-F127_\#2, respectively. However, the EE slightly decreased when the loading concentration of curcumin was increased. The emulsification and freeze drying process can promote high entrapment of the drugs in the presence of the surfactant molecules (Tahara et al., 2012; Wakabayashi et al., 2014). The zeta potential of Cur-S/W nanodispersions for cur-F68_\#2 and cur-F127_\#2 were -0.75 and 0.71, respectively.

The morphology of Cur-S/W nanodispersions was analyzed using TEM. The sizes of Cur-S/W nanodispersions measured by TEM were in good agreement with the results of DLS analysis (Figure 3). We observed spherical morphologies of Cur-S/W nanodispersions prepared with the two different types of pluronics. This finding is in contrast to that observed in our previous study, where the encapsulated CPT exhibited cubic lamellar morphologies, probably stemming from recrystallization of CPT during the formulation process because of the poor solubility of CPT in cyclohexane (Wakabayashi et al., 2014). However, here the curcumin is moderately soluble in cyclohexane, suggesting that the formation of the curcumin crystals at the early stage of preparation does not occur. The curcumin in cyclohexane is surrounded by the pluronic in water, resulting in the spherical morphologies of Cur-pluronic complexes.

\subsection{In vitro release profiles}

We investigated the in vitro release behavior of Cur-S/W nanodispersions using the dialysis method under sink condition. Figure 4 presents the percentage of curcumin release into the medium solution. We found that the free curcumin rapidly diffused into the medium within the first hour, because of the small molecular weight of curcumin $(368.38 \mathrm{Da})$. In contrast, a slower and sustained release of curcumin was found for Cur-S/W nanodispersions (Figure 4). We observed partial degradation of curcumin (approximately $20 \%$ after $350 \mathrm{~min}$.) during incubation in the release medium at $\mathrm{pH} 7.4$, possibly due to the instability of curcumin under physiological conditions (Zebib et al., 2010). Besides, in the case of Cur-S/W formulations, some part of curcumin could be retained in the dialysis bag due to the high molecular weight of pluronic. Therefore, we assumed that the equilibrium of curcumin released from Cur-S/W formulations reached $50-60 \%$ under the current experimental conditions.

The release rate of curcumin from Cur-F68 S/W nanodispersions was slightly faster than that of Cur-F127; approximately $60 \%$ and $51 \%$ of curcumin had been released into the medium from Cur-F68 and Cur-F127 S/W nanodispersions at $350 \mathrm{~min}$, respectively. These results suggested that the hydrophobicity and molecular weight of pluronic could affect the release rate of curcumin from the S/W formulations. Pluronic F68 has a higher HLB value and smaller molecular weight compared with Pluronic F127 (Table 1), leading to slightly faster release kinetics.

\subsection{Anticancer activity of Cur-S/W nanodispersions}

The in vitro anticancer activity of Cur-S/W nanodispersions was evaluated by the WST- 8 method using HeLa cells. As shown in Figure 5, the cell viability decreased with 


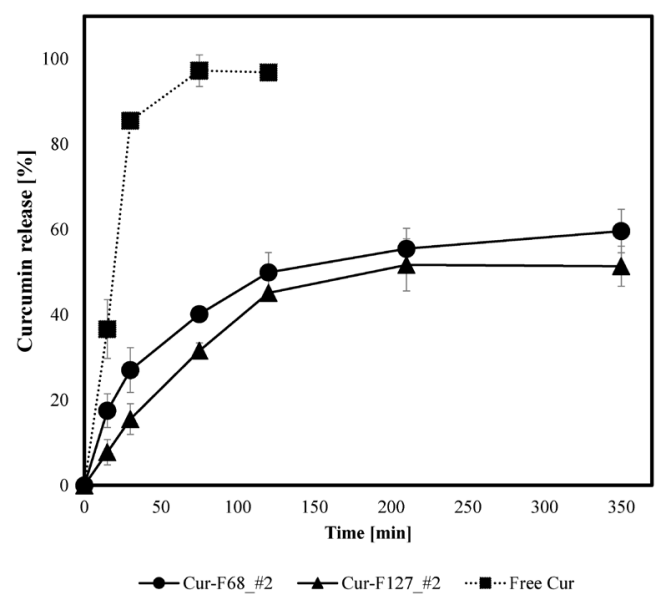

Fig. 4 In vitro release profile of free Cur and Cur-S/W nanodispersions

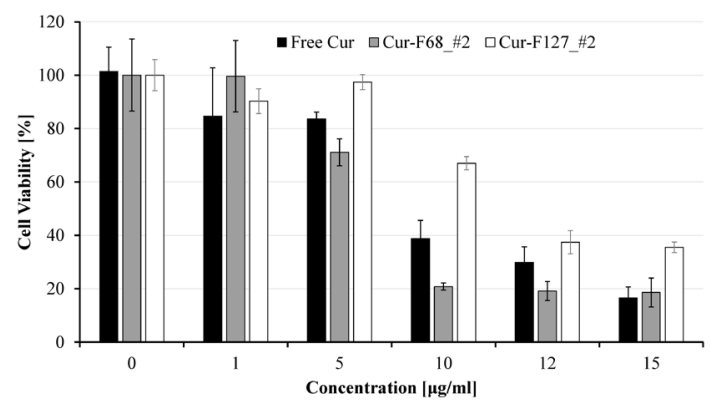

Fig. 5 Anticancer activity of free cur and Cur-S/W nanodispersions on HeLa cells with $24 \mathrm{~h}$ of incubation

increased curcumin concentration. In contrast, HeLa cells treated with the two types of pluronic as a carrier exhibited approximately $100 \%$ cell viability at concentrations up to $1000 \mu \mathrm{g} / \mathrm{mL}$, suggesting that the carrier has no significant effects on the cell viability of HeLa cells (Figure S1). The non-toxic effect of the carrier was also noticed with the cell viability of normal cells (NIH3T3 cell line) at concentrations up to $2000 \mu \mathrm{g} / \mathrm{mL}$ (Figure S2).

Free curcumin showed a half maximal inhibitory concentration $\left(\mathrm{IC}_{50}\right.$ ) value of $\sim 10 \mu \mathrm{g} / \mathrm{mL}$, which is consistent with the value reported previously (Razi et al., 2018a). We found a clear difference between free Cur and Cur-S/W formulation (Figure 5). For examples with the curcumin concentration of $10 \mu \mathrm{g} / \mathrm{mL}$, the anticancer activity of Cur-F68 S/W nanodispersion was higher than that of Cur-F127 formulation and free curcumin, and free Cur was higher than that of the Cur-F127 S/W nanodispersions. The difference in anticancer activity of the Cur-S/W nanodispersions with two different types of pluronic might correlate to the release profile (Figure 4) and the cellular uptake profile of the Cur-S/W formulations (Figure 5).

\subsection{Cellular internalization of Cur-S/W nanodispersion}

We evaluated the cellular internalization of Cur-S/W nanodispersions with two different surfactants using CLSM

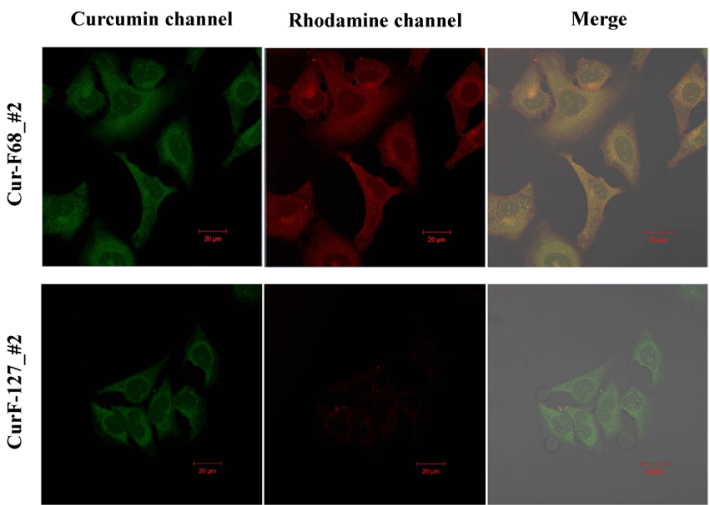

Fig. 6 Confocal images of HeLa cells treated with Cur-S/W nanodispersions for $3 \mathrm{~h}$

(Figure 6). Since the curcumin is auto-fluorescent, it could be directly observed using a FITC filter, as green fluorescence. Moreover, the Rho-DOPE was incorporated with Cur-S/W nanodispersions for tracking the particle complex on the cells. The cellular uptake of Cur-S/W nanodispersion was observed after $3 \mathrm{~h}$ of incubation. Figure 6 shows that the green fluorescence derived from curcumin was located in the cytoplasm of HeLa cells, where the intensity of green fluorescence observed in the cells after treatment with Cur-F68 S/W nanodispersion was slightly higher than those treated with Cur-F127 S/W nanodispersion. In addition, stronger red fluorescence from Rho-DOPE was detected in the cells treated with Cur-F68 S/W nanodispersion compared with Cur-F127 S/W nanodispersion, indicating that the cellular internalization of Cur-F68 S/W was higher than Cur-F127 S/W nanodispersion. Cur-S/W nanodipersions were prepared as previously reported (Wakabayashi et al., 2014), by which the hydrophobic molecule was incorporated into the hydrophobic block PPO of pluronic and the hydrophilic block PEO tails directed to the aqueous solution. We could assume that the cellular internalization of Cur-S/W nanodisperisons was facilitated by the endocytic pathway as observed in our previous study with the same pluronicbased S/W nanodispersions, given that the basic structure of $\mathrm{S} / \mathrm{W}$ nanodispersions for the solubilization of hydrophobic molecules was the same (Wakabayashi et al., 2014).

The highest cellular uptake of the complex may be at tributed to the higher cytotoxicity on the HeLa cells (Figure 5). It has been previously reported that more hydrophobic nanocarriers prepared with Pluronic F127 than those with F68 showed slightly higher cellular internalization because of the higher affinity for the cellular membrane (Kim et al., 2010). However, apart from the hydrophobicity of pluronic surfactants, the length of the hydrophilic block PEO chain of the pluronic nanocarrier also has an influence on the cellular uptake (Arranja et al., 2016). The longer PEO block in nanocarriers may result in more extensive hydration and less exposure of the hydrophobic core. Here, Pluronic F68 had a shorter hydrophilic block PEO compared with F127 (average number of EO units for Pluronic F68 and F127 are 76 and 100, respectively). Moreover, the particle size of Cur- 
F127 S/W nanodispersions is much smaller than that of Cur-F68 S/W nanodispersions, implying a packed arrangement of the pluronic surfactant on the surface. These may result in the lower accessibility of the Cur-F127 nanocarrier to the cellular membrane. The slightly slower release kinetics of Cur-F127 suggests the superior stability of the nanocarrier and further supports this hypothesis. Taken together, the higher cellular internalization and the slightly higher release profile of Cur-F68 S/W nanocarriers resulted in the higher anticancer activity observed.

\section{Conclusions}

Curcumin was successfully encapsulated with high entrapment efficiency using the $\mathrm{S} / \mathrm{W}$ nanodispersion technique. The enhanced water solubility of curcumin was achieved by the encapsulation of nonionic surfactant pluronic with the nanosized particles of curcumin in aqueous solution. In vitro release studies showed that the release rate of curcumin from the resultant complexes could be controlled by choosing a proper surfactant. A correlation between the release profile of the Cur-S/W nanodispersions and anticancer activity was observed. The improved water solubility of curcumin and observed anticancer activity of the Cur-S/W nanodispersions in this study have thus increased the potential of curcumin in pharmaceutical applications.

\section{Supplementary Information}

Supplementary Information is available at http://www.scej.org/publication/jcej/suppl/

\section{Acknowledgement}

This work was supported by a Grant-in-Aid for Scientific Research (S) from the Ministry of Education, Cultures, Sports, Science and Technology (MEXT) of Japan (Grant Number JP16H06369). MEXT scholarship provided to S.D.H (Scholarship ID No. 153596) is gratefully acknowledged.

We thank Amy Tong from Edanz Group (www.edanzediting.com/ac) for editing a draft of this manuscript.

\section{Literature Cited}

Anand, P., A. B. Kunnumakkara, R. A. Newman and B. B. Aggarwal; "Bioavailability of Curcumin: Problems and Promises," Mol. Pharm., 4, 807-818 (2007)

Arranja, A., A. G. Denkova, K. Morawska, G. Waton, S. Van Vlierberghe, P. Dubruel, F. Schosseler and E. Mendes; "Interactions of Pluronic Nanocarriers with 2D and 3D Cell Cultures: Effects of PEO Block Length and Aggregation State," J. Control. Release, 224, 126-135 (2016)

Bayet-Robert, M., F. Kwiatkowski, M. Leheurteur, F. Gachon, E. Planchat, C. Abrial, M. A. Mouret-Reynier, X. Durando, C. Barthomeuf and P. Chollet; "Phase I Dose Escalation Trial of Docetaxel Plus Curcumin in Patients with Advanced and Metastatic Breast Cancer," Cancer Biol. Ther., 9, 8-14 (2010)

Chang, C., T. Wang, Q. Hu, M. Zhou, J. Xue and Y. Luo; "Pectin Coating
Improves Physicochemical Properties of Caseinate/zein Nanoparticles as Oral Delivery Vehicles for Curcumin," Food Hydrocoll., 70, 143-151 (2017)

Chen, L. C., Y. C. Chen, C. Y. Su, W. P. Wong, M. T. Sheu and H. O. Ho; "Development and Characterization of Lecithin-Based SelfAssembling Mixed Polymeric Micellar (saMPMs) Drug Delivery Systems for Curcumin," Sci. Rep., 6, 1-12 (2016)

Gong, C., S. Deng, Q. Wu, M. Xiang, X. Wei, L. Li, X. Gao, B. Wang, L. Sun, Y. Chen, Y. Li, L. Liu, Z. Qian and Y. Wei; "Improving Antiangiogenesis and Anti-Tumor Activity of Curcumin by Biodegradable Polymeric Micelles," Biomaterials, 34, 1413-1432 (2013)

Gupta, S. C., S. Patchva and B. B. Aggarwal; "Therapeutic Roles of Curcumin: Lessons Learned from Clinical Trials," AAPS J., 15, 195-218 (2013)

Kim, J. Y., W. Choi, Y. H. Kim, G. Tae, S. Y. Lee, K. Kim and I. C. Kwon; "In-vivo Tumor Targeting of Pluronic-Based Nano-Carriers," J. Control. Release, 147, 109-117 (2010)

Lao, C. D., M. T. Ruffin 4th, D. Normolle, D. D. Heath, S. I. Murray, J. M. Bailey, M. E. Boggs, J. Crowell, C. L. Rock and D. E. Brenner; "Dose Escalation of a Curcuminoid Formulation. BMC Complement," Altern. Med, 6, 10-14 (2006)

Li, L., F. S. Braiteh and R. Kurzrock; "Liposome-Encapsulated Curcumin: In Vitro and In Vivo Effects on Proliferation, Apoptosis, Signaling, and Angiogenesis," Cancer, 104, 1322-1331 (2005)

Mathew, D. and W. L. Hsu; "Antiviral Potential of Curcumin," J. Funct. Foods, 40, 692-699 (2018)

Razi, M. A., R. Wakabayashi, Y. Tahara, M. Goto and N. Kamiya; "Genipin-Stabilized Caseinate-Chitosan Nanoparticles for Enhanced Stability and Anti-cancer Activity of Curcumin," Colloids Surf. B Biointerfaces, 164, 308-315 (2018a)

Razi, M. A., R. Wakabayashi, M. Goto and N. Kamiya; "Formation and Characterization of Caseinate-Chitosan Nanocomplexes for Encapsulation of Curcumin," J. Chem. Eng. Japan, 51, 445-453 (2018b)

Sari, T. P., B. Mann, R. Kumar, R. R. B. Singh, R. Sharma, M. Bhardwaj and S. Athira; "Preparation and Characterization of Nanoemulsion Encapsulating Curcumin," Food Hydrocoll., 43, 540-546 (2015)

Sezgin, Z., N. Yüksel and T. Baykara; "Preparation and Characterization of Polymeric Micelles for Solubilization of Poorly Soluble Anticancer Drugs," Eur. J. Pharm. Biopharm., 64, 261-268 (2006)

Tadros, T.; "Polymeric Surfactants in Disperse Systems," Adv. Colloid Interface Sci., 147-148, 281-299 (2009)

Tahara, Y., N. Kamiya and M. Goto; "Solid-in-Oil Dispersion: A Novel Core Technology for Drug Delivery Systems," Int. J. Pharm., 438, 249-257 (2012)

Tønnesen, H. H., M. Másson and T. Loftsson; "Studies of Curcumin and Curcuminoids. XXVII. Cyclodextrin Complexation: Solubility, Chemical and Photochemical Stability," Int. J. Pharm., 244, 127-135 (2002)

Wakabayashi, R., R. Ishiyama, N. Kamiya and M. Goto; "A Novel Surface-coated Nanocarrier for Efficient Encapsulation and Delivery of Camptothecin to Cells," MedChemComm, 5, 1515-1519 (2014)

WHO; Global Health Estimates 2000-2015, Deaths by Cause, Age and Sex (2017).

Yang, X., Z. Li, N. Wang, L. Li, L. Song, T. He, L. Sun, Z. Wang, Q. Wu, N. Luo, C. Yi and C. Gong; "Curcumin-Encapsulated Polymeric Micelles Suppress the Development of Colon Cancer In Vitro and In Vivo," Sci. Rep., 5, 1-15 (2015)

Zebib, B., Z. Mouloungui and V. Noirot; "Stabilization of Curcumin by Complexation with Divalent Cations in Glycerol/Water System," Bioinorg. Chem. Appl., 292760 (2010) 